\title{
Apriori-LLR-Threshold-Assisted $K$-Best Sphere Detection for MIMO Channels
}

\author{
Li Wang, Lei Xu, Sheng Chen and Lajos Hanzo \\ School of ECS, University of Southampton, SO17 1BJ, UK. \\ Tel: +44-23-8059 3125, Fax: +44-23-8059 4508 \\ Email: $\{1 w 05 r, 1 x 04 r$, sqc, lh\}@ecs.soton.ac.uk; http://www-mobile.ecs.soton.ac.uk
}

\begin{abstract}
When the maximum number of best candidates retained at each tree search level of the $K$-Best Sphere Detection (SD) is kept low for the sake of maintaining a low memory requirement and computational complexity, the SD may result in a considerable performance degradation in comparison to the full-search based Maximum Likelihood (ML) detector. In order to circumvent this problem, in this contribution we propose a novel complexity-reduction scheme, referred to as the Apriori-LLRThreshold (ALT) based technique for the $K$-best SD, which was based on the exploitation of the $a$ priori LLRs provided by the outer channel decoder in the context of iterative detection aided channel coded systems. For example, given a BER of $10^{-5}$, a nearML performance is achieved in an $(8 \times 4)$-element rank-deficient 4-QAM system, despite imposing a factor two reduced detection candidate list generation related complexity and a factor eight reduced extrinsic LLR calculation related complexity, when compared to the conventional SD-aided iterative benchmark receiver. The associated memory requirements were also reduced by a factor of eight.
\end{abstract}

\section{Motivation}

Although the classic Maximum A Posteriori (MAP) detector offers tremendous performance advantages over sub-optimum detection methods in a channel coded Multiple-Input MultipleOutput (MIMO) system, it exhibits a potentially excessive computational complexity, especially in high-throughput systems invoking high-order modulation schemes or a high number of transmit antennas. The family of sphere detection (SD) arrangements $[1,2]$ constitutes the most promising lowcomplexity near-ML detection class and hence its representatives have recently attracted substantial research attention. Amongst the different SD techniques, the $K$-best SD [2] has several advantages over its so-called depth-first counterpart [1], such as for example imposing a fixed detection complexity and a flexible hardware implementation. However, the complexity imposed by the $K$-best SD becomes also high in heavily rank-deficient MIMO systems, where the number of tansmit antennas exceeds that of the receive antennas, such as our $(8 \times 4)$-element system, if a near-MAP performance is expected. In order to enable the $K$-best SD to achieve a near-MAP performance at an affordable complexity in such challenging scearios, the novel contribution of this treatise is that we exploit the a priori LLRs provided by the outer channel decoder for controlling the detector's performance

Acknowledgements: The work reported in this paper has formed part of the Core 4 Research Programme of the Virtual Centre of Excellence in Mobile and Personal Communications, Mobile VCE, www.mobilevce.com, whose funding support, including that of EPSRC, is gratefully acknowledged. Fully detailed technical reports on this research are available to Industrial Members of Mobile VCE. versus complexity trade-off. We refer to this detector as the Apriori-LLR-Threshold (ALT) aided SD technique.

The rest of this paper is organised as follows. Section II describes the model of our Multiple-Input Multiple-Output (MIMO) aided OFDM system. After a brief review over the $K$-best list SD, the proposed ALT based technique and the corresponding receiver architecture are presented in Section III. Our simulation results and conclusions are provided in Section IV and Section V, respectively.

\section{SySTEM MODEL}

Consider a spatial division multiplexing (SDM) aided orthogonal frequency division multiplexing (OFDM) system equipped with $M$ uplink transmit antennas and $N$ receiver antennas [3]. The subcarrier-related equivalent basedband model of the MIMO-OFDM system is given by [3]:

$$
\mathbf{y}=\mathbf{H s}+\mathbf{w}
$$

where $\mathbf{y} \in \mathbb{C}^{N \times 1}, \mathbf{s} \in \mathbb{C}^{M \times 1}$ and $\mathbf{w} \in \mathbb{C}^{N \times 1}$ denote the received and transmitted signal vectors as well as the AWGN sample vector having a variance of $\sigma_{w}^{2}$ per dimension, respectively. Moreover, $\mathbf{H}$ is a $(N \times M)$-element frequency-domain channel transfer factor (FDCHTF) matrix, where each column represents the unique spatial signature of the corresponding transmit antenna. Here, we assume that the FDCHTFs are independent, stationary, complex valued Gaussian distributed processes with a zero-mean and a unit variance [3].

\section{Apriori-LLR-THRESHOLD-ASSISTED LOW-COMPLEXITY $K$-BEST SD}

\section{A. K-Best List Sphere Detection}

With the aid of the unconstrained MMSE solution of $\hat{\mathbf{x}}_{c}=$ $\left(\mathbf{H}^{H} \mathbf{H}+\sigma_{w}^{2} \mathbf{I}\right) \mathbf{H}^{H} \mathbf{y}$ and the Cholesky factorization [1], the well-known ML solution can be formulated as:

$$
\hat{\mathbf{s}}_{M L}=\arg \min _{\check{\mathbf{s}} \in M_{c}^{M}}|| \mathbf{y}-\mathbf{H} \check{\mathbf{s}} \|_{2}^{2},
$$

where $M_{c}$ is the set of modulated symbol points in the constellation and $M$ is the number of uplink (UL) transmit antennas employed by the system. It can be shown the Eq.(2) may be expressed as [4]:

$$
\begin{gathered}
\hat{\mathbf{s}}_{M L}=\arg \min _{\check{\mathbf{s}} \in M_{c}^{M}}\left(\check{\mathbf{s}}-\hat{\mathbf{x}}_{c}\right)^{H} \mathbf{U}^{H} \mathbf{U}\left(\check{\mathbf{s}}-\hat{\mathbf{x}}_{c}\right), \\
=\arg \min _{\check{\mathbf{s}} \in M_{c}^{M}} \sum_{i=1}^{M} \underbrace{u_{i i}^{2}\left[\check{s}_{i}-\hat{x}_{i}+\sum_{j=i+1}^{M} \frac{u_{i j}}{u_{i i}}\left(\check{s}_{j}-\hat{x}_{j}\right)\right]^{2}}_{\phi},(4)
\end{gathered}
$$


where $\mathbf{U}$ is a $(M \times M)$-element upper-triangular matrix, which satisfies $\mathbf{U}^{H} \mathbf{U}=\mathbf{H}^{H} \mathbf{H}+\sigma_{w}^{2} \mathbf{I}_{\mathcal{C}}$. For the $K$-best SD [2], instead of expanding every node at each tree search level, we only retain a fixed number of $K$ nodes, namely those having the smallest accumulated Partial Euclidean Distances (PEDs) corresponding to the term $\phi$ of Eq.(4). Hence, after the search reaches the tree leaf level, a candidate list $\mathcal{L}$ is generated, which contains $N_{\text {cand }}=K$ number of candidate solutions, which are then forwarded for extrinsic LogLikelihoold-Ratio (LLR) calculation to the iterative Soft-InputSoft-Output (SISO) receiver according to [5].

\section{B. Principle of the Apriori-LLR-Threshold Technique}

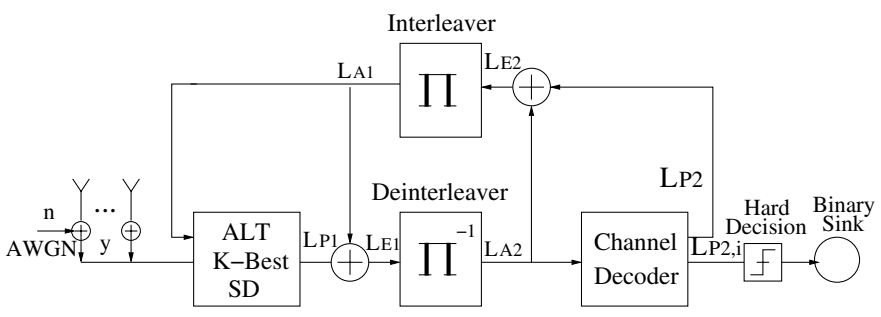

Fig. 1. ALT-assisted $K$-best SD aided iterative receiver architecture

The SD-aided iterative receiver using the proposed aprioriLLR-threshold (ALT) technique is portrayed in Figure 1, where the interleaver and deinterleaver pair seen at the receiver side divides the receiver into two parts, namely the inner SD and the outer channel decoder. Note that in Figure $1 L_{A}, L_{E}$ and $L_{D}$ denote the a priori, the extrinsic and the a posteriori LLRs, while the subscript ' 1 ' and ' 2 ' represent the bit LLRs associated with the inner detector and outer channel decoder, respectively. First of all, let us review the definition of the a priori LLRs, which is the logarithm of the ratio of the bit probabilities associated with +1 and -1 [6], that can be expressed as follows:

$$
L_{A}\left(b_{j}\right)=\ln \frac{P\left[b_{j}=+1\right]}{P\left[b_{j}=-1\right]} .
$$

Therefore, the sign of the resultant LLRs indicates whether the current bit is more likely to be +1 or -1 , whereas the magnitude reflects how reliable the decision concerning the current bit is. For example, given a large positive a priori LLR delivered by the outer channel decoder of Figure 1, it is inferred that the corresponding transmitted bit is likely to have been +1 . In light of this, the search tree of the $K$ best SD of Section III-A is depicted in Figure 2(a), which may be significantly simplified by invoking an ALT controlled technique. To be specific, first we consider a BPSK modulated four-transmit-antenna scenario as an example, where we have a detection list size of $N_{\text {cand }}=K=2$, which means that the two best candidates corresponding to the lowest two acculated PEDs are retained at each search tree level. If the a priori LLR of the $m$ th transmit antenna's BPSK symbol is sufficiently high, then there is no need to continue the tree-search for that particular antenna during the SD process. In other words, at the $m$ th tree search level, we retain a single branch instead of retaining both legitimate detection options.

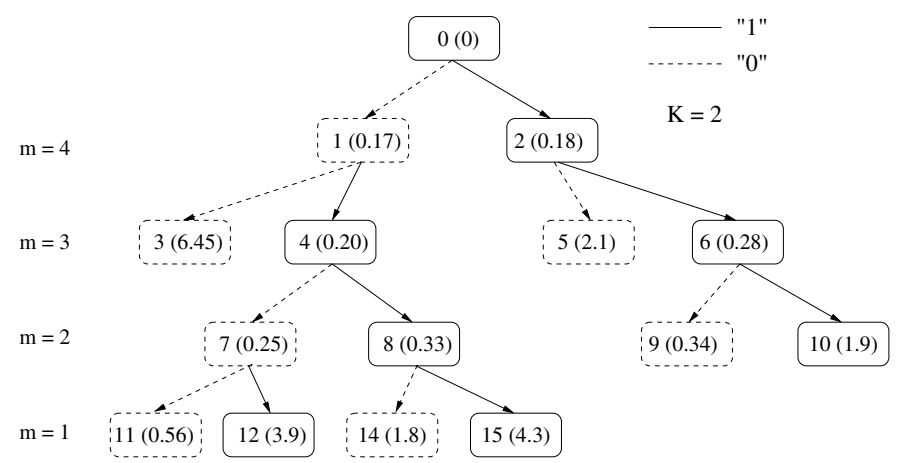

(a) The search tree of the $K$-Best SD dispensing with ALT in the Scenario of a four-transmit-antenna BPSK SDM system, where $K=2$ and the $T_{A L T}=7$

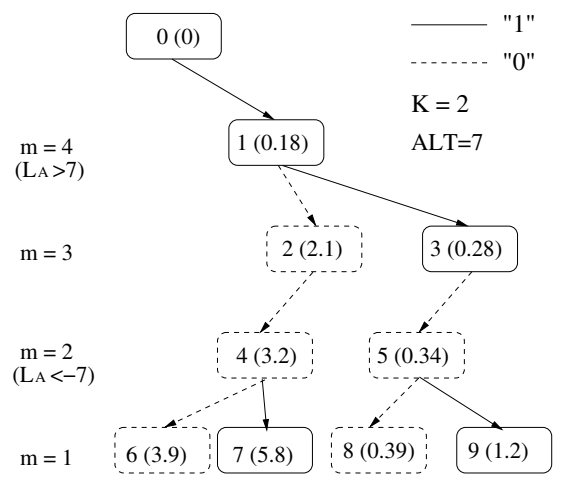

(b) The search tree of the ALT-assisted $K$-best SD in the Scenario of a four-transmit-antenna BPSK SDM system, where $K=2$ and the $T_{A L T}=7$

Fig. 2. Illustration of the $K$-best SD algorithm with the aid of ALT scheme where $T_{A L T}=7$ : The figure in () indicates the PED of a specific node for the trial point in the modulated constellation; while the number outside represents the order in which the points are visited.

More explicitly, as seen in Figure 2(a), when dispensing with the ALT scheme, after 15 PED evaluations the SD opts for the specific tree leaf having a Euclidean distance of 0.56 . Then the search portrayed in Figure 2(a) backtracks to the $(m=4)$ th level, yielding the hypothesized ML solution. However, as the ALT based scheme is invoked, a pruned search tree is obtained for the $K$-best $\mathrm{SD}$, which is shown in Figure 2(b), if we assume that the absolute values of the a priori LLRs of both the $(m=4)$ th and the $(m=2)$ nd transmit antennas exceed the preset ALT value of $T_{A L T} 7$ after a certain number of iterations between the SD and the channel decoder. Specifically, the SD will discard all the branches corresponding to $s_{4}=0$ at the $(m=4)$ th level and $s_{2}=1$ at the $(m=2)$ nd level. Consequently, only $N_{P E D}=9$ PED evaluations have been carried out for this particular example, indicating a considerable reduction of the computational complexity imposed. However, even more importantly, the SD successfully identified the true ML solution, which is different from the one generated by the SD characterized in Figure 2(a), which dispensed with the ALT based technique. This was achieved by backtracking during the search from a different tree leaf having a smaller Euclidean distance of 0.39 to the $(m=4)$ th level. Thus the incorrect search branch corresponding to $s_{4}=0$ was discarded as early as at the $(m=4)$ th tree level, hence reducing the computational complexity imposed, while simultaneously avoiding the situation of discarding a potential path leading to 
the true ML solution. By contrast, the potential ML solution may be discarded by the $K$-best SD using no thresholding at the $(m=2)$ nd level due to the fact that the true ML path may have a temporarily larger PED. Thus, in addition to the achievable complexity reduction, the main benefit of employing the ALT based scheme for the $K$-best SD is its potential performance improvement. Although the number of candidates retained at the $m$ th search tree level of Figure 2 remains $K$, the affordable search-complexity is specifically focused on the candidates having a specific bit value at the $m$ th position, which is determined by the proposed LLR-based decision. In other words, the LLR-based search-tree pruning portrayed in Figure 2(b) decreases the probability of discarding a potentially correct path at an early search stage, especially when the value of $K$ is set relatively small in the interest of maintaining a low complexity.

\section{Choice of the LLR Threshold}

Previously, we have assumed an LLR threshold of $T_{A L T}=$ 7 , which ensured that the proposed ALT scheme performed well. However, it is intuitive that the LLR threshold cannot be set arbitrarily, since it plays a vital role in determining the system's performance. To be more specific, if the threshold is set too high, the ALT scheme hardly affects the system's operation, since the a priori LLRs provided by the outer decoder are unlikely to be higher than the threshold, even after several iterations. By contrast, if the threshold is set to an excessively low value, though the computational complexity can be substantially reduced, naturally, a BER performance degradation is imposed. The above conjectures are verified by Figure 3, where the bars in the histogram of Figure 3(a) represent the computational complexity imposed, which was quantified in terms of the number of the PED-evaluations per channel use. The LLR-thresholds employed by the ALTassisted K-Best SD were set to values of $T_{A L T}=4,7$ and 10 , indicating that the lower the LLR-threshold, the higher the complexity reduction attained. The corresponding BER curves plotted in Figure 3(b), however, demonstrate that when the threshold is set to an excessively low value, this may be expected to impose a performance degradation, as the SNR increases. This is not unexpected because when the SNR becomes high, the a priori LLRs fed back by the outer decoder to the SD are becoming predominantly higher than the LLRthreshold set at the very beginning of the iterative detection process. This may trigger an aggressive search-tree-truncation, which in turn results in discarding the true ML branch. In other words, in this scenario the truncation introduced by the ALT technique was activated too early, before the receiver attained a sufficiently high iterative gain. For example, given a target BER of $10^{-5}$, a performance gain of about $1.5 \mathrm{~dB}$ was observed in Figure 3(b) over that of the receiver operating without the ALT technique, with the aid of a threshold of $T_{A} L T=7$, while a performance degradation of about $1.5 \mathrm{~dB}$ was imposed by setting the threshold to $T_{A L T}=4$. Note in Figure 3(b) that the BER curve corresponding to the threshold of $T_{A L T}=10$ is actually coincident with that of the 'non-ALTassisted' system, as shown in Figure 4, implying that the ALT scheme does not have any beneficial effect with the aid of

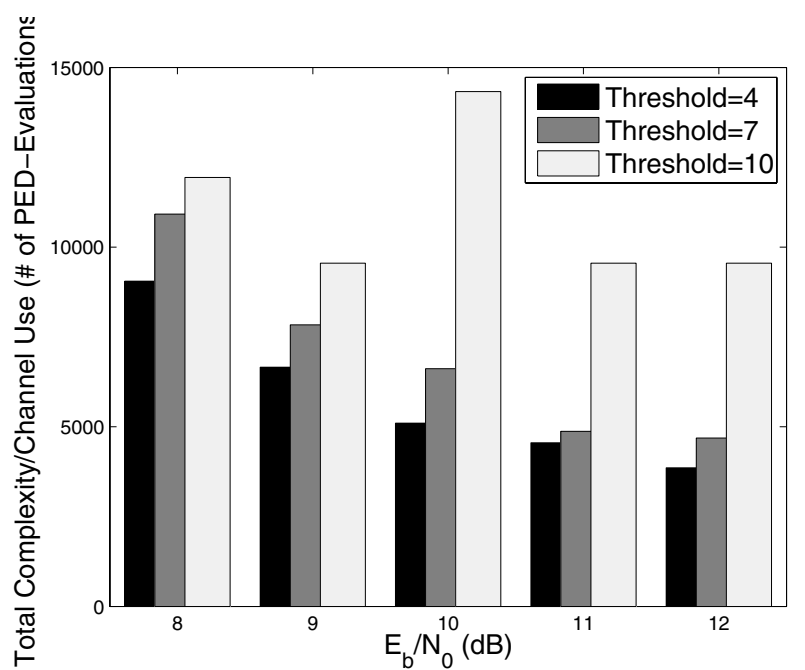

(a) Effects on computational complexity $(K=128)$

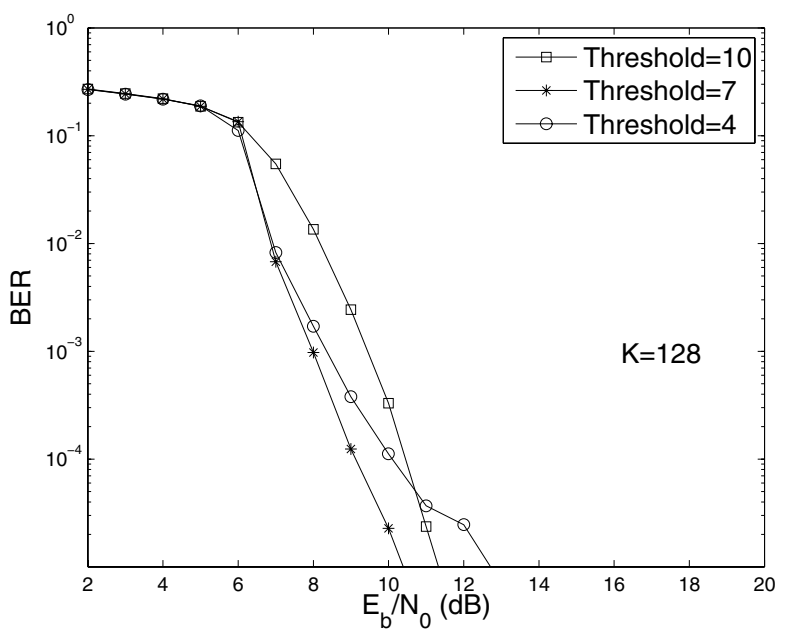

(b) Effects on BER performance $(K=128)$

Fig. 3. Effects of the LLR-threshold on both the BER performance and the computational compleixty of the $K$-best SD iterative receiver in an $(8 \times 4)$ element 4QAM SDMA/OFDM System. All other system parameters are listed in Table I.

such a high threshold value. In conclusion, the threshold has to be carefully adjusted for the sake of achieving the target performance as a function of the SNR encountered.

\section{Simulation Results And Discussion}

\section{A. BER Performance}

Figure 4 depicts the BER performance of the ALT-assited $K$-best SD in the scenario of an $(8 \times 4)$-element rankdeficient 4-QAM SDM/OFDM system in comparison to the system dispensing with the ALT technique. Given a target BER of $10^{-5}$ and a fixed list-length of $\mathcal{N}_{\text {cand }}=K=16$, a performance gain of $2.5 \mathrm{~dB}$ can be achieved by setting the $a$ priori-LLR-threshold to $T_{A L T}=7$, which is reduced to about $1 \mathrm{~dB}$ for the system using $K=128$. Actually, the ALT-aided receiver associated with $K=128$ has already approached the MAP performance, which required at least $K=1024$ for the equivalent system dispensing with the ALT technique. The ratio of these system complexities associated with the extrinsic-LLR-calculation is as high as eight. 


\begin{tabular}{|c|c|}
\hline System Parameters & Choice \\
\hline System & SDM/OFDM \\
\hline No. of Sub-Carriers & 128 \\
\hline Modulation & 4-QAM \\
\hline No. of Transmit Antenna & 8 \\
\hline No. of Receive Antenna & 4 \\
\hline Block Length & 10240 \\
\hline CIR Model & $P\left(\tau_{k}\right)=\left[\begin{array}{lll}0.5 & 0.3 & 0.2\end{array}\right],(k=0,1,2)$ \\
\hline CIR Tap Fading & OFDM symbol invariant \\
\hline Channel Estimation & Ideal \\
\hline Detector/MAP & $K$-Best List-SD \\
\hline List Length $\mathcal{N}_{\text {cand }}$ & $=K$ \\
\hline \multirow{3}{*}{ Channel Encoder } & $\operatorname{RSC}(2,1,3)$ \\
\hline & Generator Polynomials $(6 / 13)$ \\
\hline & Code Termination (Off) \\
\hline No. of Iterations (Variable) & $\begin{array}{l}\text { Iterations terminate as soon as the } \\
\text { resultant trajectory line reaches the } \\
\text { convergence point or became trapped }\end{array}$ \\
\hline
\end{tabular}

TABLE I

Summary of System Parameters For the K-Best SD Aided Coded SDM/SDMA OFDM SYSTEM

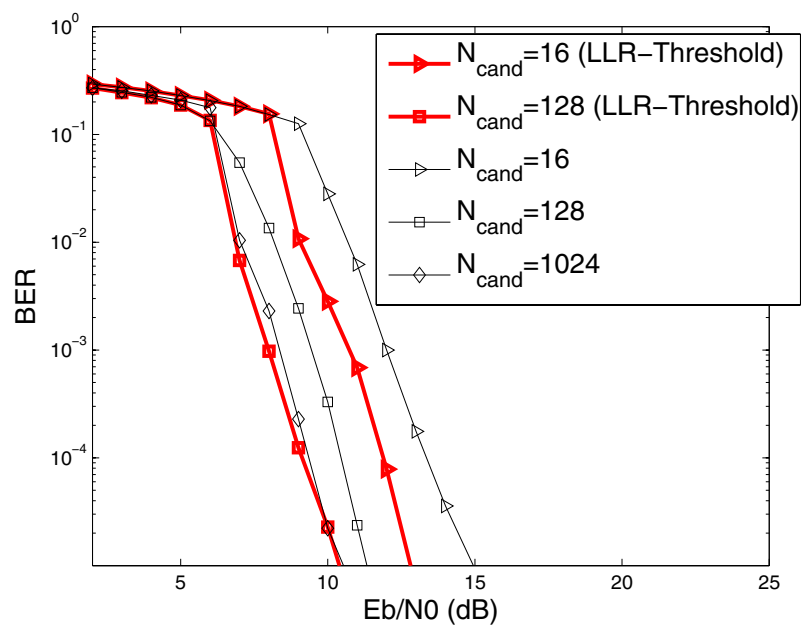

Fig. 4. BER performance of the two-stage LLR-threshold-aided $K$-best SD $\left(T_{A L T}=7\right)$ iterative receiver in an $(8 \times 4)$-element 4QAM SDM/OFDM System. All other system parameters are listed in Tabel I

\section{B. Computational Complexity}

The complexity imposed by invoking the ALT scheme can be viewed in Figure 5(a), where the overall computational complexity quantified in terms of the number of PEDevaluations per channel use imposed by the system operating both with and without the aid of the ALT scheme are plotted for the $(8 \times 4)$-element rank-deficient 4QAM SDM/OFDM system scenario. Specifically, since SD not benefitting from the ALT technique has to be carried out only once per channel use, regardless of how many iterations are used, it exhibits the same computational complexity of 2,388 PED-evaluations per channel use for $K=128$, regardless of the channel SNR. By contrast, the number of PED-evaluations required by the ALT-assisted receiver differs for different SNRs, since the candidate-list has to be regenerated by the ALT-aided SD at each iteration. To be specific, observe in Figure 5(a) that the complexity increases steadily as the SNR increases from $2 \mathrm{~dB}$ to $7 \mathrm{~dB}$, peaking at about $17,000 \mathrm{PED}$-evaluations per channel use. Beyond $7 \mathrm{~dB}$, the complexity decays steadily as the SNR increases further, but levels out around 5,000 PED-evaluations at an SNR of $12 \mathrm{~dB}$. With reference to Figure 5(a), the candidate-list-generation complexity of the ALT-aided receiver

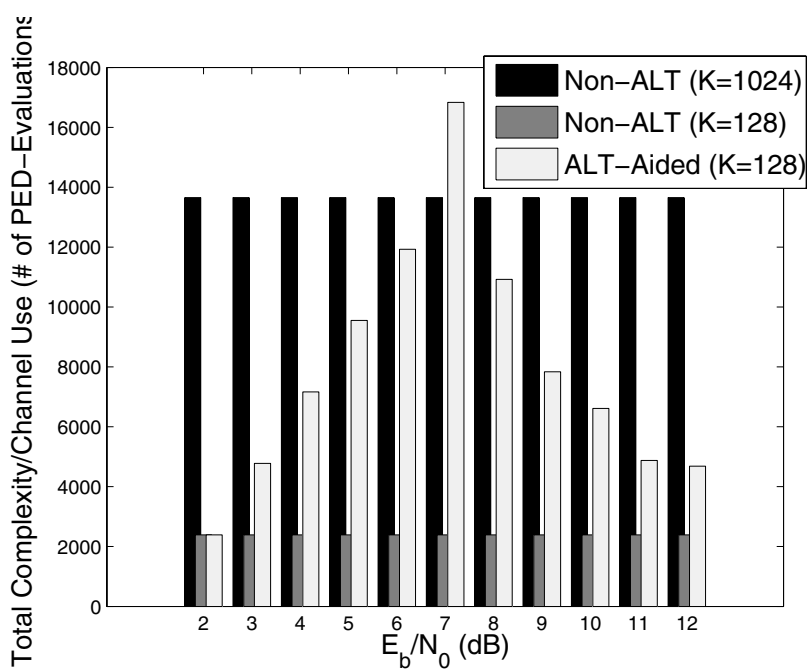

(a)

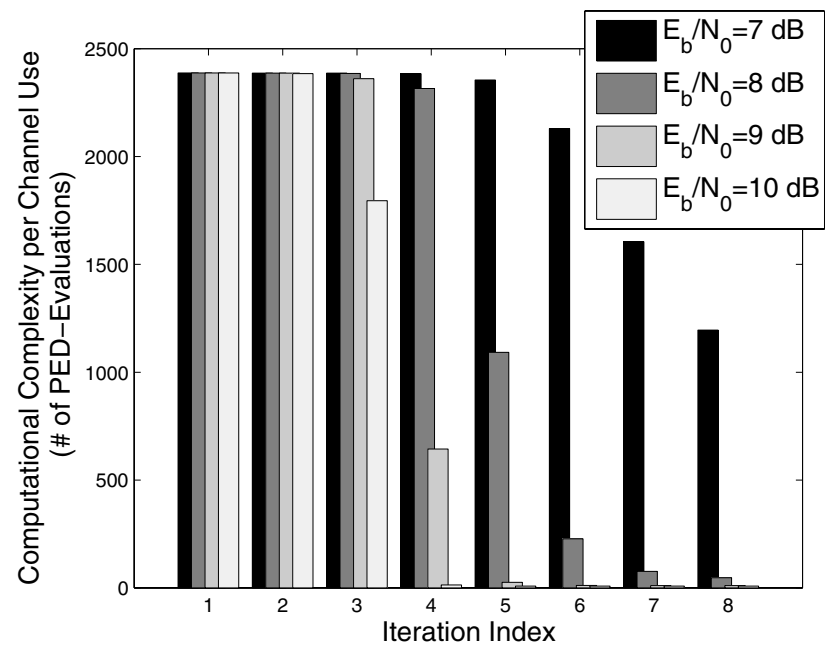

(b)

Fig. 5. Histogram of the candidate-list-generation-related computational complexity imposed by the ALT-aided $K$-best SD: (a) the overall computational complexity per channel use for different $E_{b} / N_{0}$ values; (b) computational complexity per channel use of each iteration $(\mathrm{K}=128)$. All other system parameters are listed in Table I.

is well below that of its 'non-ALT-aided' counterpart for the SNR range spanning from $2 \mathrm{~dB}$ to $12 \mathrm{~dB}$ except for SNRs in the immediate vicinity of $7 \mathrm{~dB}$, if our aim is to achieve the near-MAP BER performance quantified in Figure 4. Recall that such a high performance can only be attained by having $K=N_{\text {cand }}=1024$ for the system operating without the ALT technique or by setting $K=N_{\text {cand }}=128$ in the presence of the ALT scheme. Therefore, in the presence of the ALT scheme, the candidate list has to be regenerated at each iteration. Nontheless, the total complexity imposed is substantially reduced, except for SNRs in the immediate vicinity of $7 \mathrm{~dB}$.

Furthermore, when the SNR is high, the number of PEDevaluations carried out at each iteration is expected to decrease, as the iterations continue, as oberved in Figure 5(b). This is due to the fact that the a priori LLRs fed back from the outer decoder to the SD are likely to become higher than the LLR threshold after the first few iterations, and this allows the ALT- 


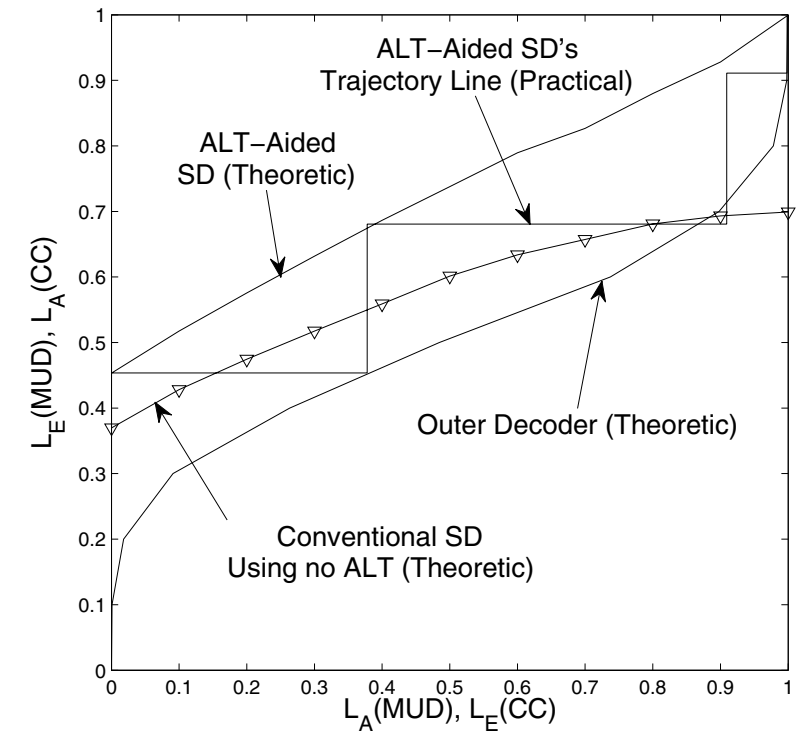

Fig. 6. EXIT chart of the ALT-aided $K$-Best SD $(K=128)$ iterative receiver in the scenario of an $(8 \times 4)$-element 4-QAM SDM/OFDM system at $\mathrm{SNR}=10 \mathrm{~dB}$. All other system parameters are listed in Table I.

assisted SD to directly truncate the low-probability branches, hence leading to a reduced multi-user/mutli-antenna constellation size, which in turn results in a reduced complexity.

\section{EXIT Chart Analysis}

The performance gain achieved by the employment of the ALT technique observed in Figure 4, can be interpreted with the aid of the corresponding EXIT chart [7] portrayed in Figure 6. Specifically, the resultant EXIT curve of the ALTaided SD is evidently above that of the SD dispending with ALT, leading to the benefit of attaining a higher iteration gain with less iterations.

\section{Effects of Non-Gaussian Distributed LLRs Imposed by the ALT Technique}

In order to investigate the reason behind the EXIT chart mismatch seen in Figure 6, we refer to the histograms of both the a priori and the extrinsic LLRs of the ALT-aided SD in Figure 7. Recall that the EXIT chart analysis of an iterative receiver is sufficiently accurate only on condition, when the a priori LLRs at the input and the a posteriori LLRs at the output of a constituent module of the iterative scheme exhibit a Gaussian distribution. However, we found that the application of the ALT scheme actually degrades the accuracy of the approximate Gaussian distribution exhibited by the LLRs at an early stage of the iterations, resulting in a severe EXIT chart mismatch problem. Hence, although the theoretical EXIT curve of the ALT-aided receiver obtained under the assumption of having near-Gaussian distributed LLRs all the time can indeed reach the $(1,1)$ point of Figure 6 , only a limited maximum iterative gain can be achieved because the resultant decoding trajectory gets trapped before reaching the point of perfect convergence, as seen in Figure 6. Hence, a residual error floor persists.
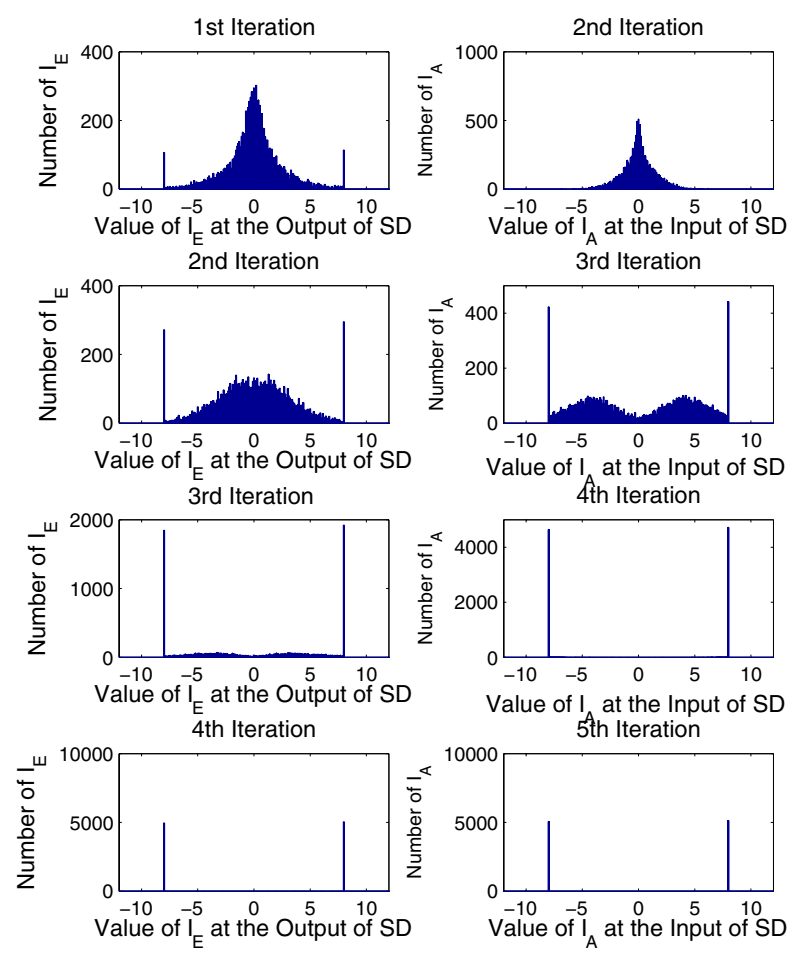

Fig. 7. Histogram of the LLRs at both the input and the output of the ALT-assisted $K$-best SD during the iterative process in the scenario of an $(8 \times 4)$-element 4-QAM SDM/OFDM system for $K=N_{\text {cand }}=128$, ALTaided, $\mathrm{SNR}=10 \mathrm{~dB}$.

\section{CONCLUSION}

A novel Apriori-LLR-Threshold-aided SDs was proposed, which is capable of achieving a near-MAP performance at a significantly reduced complexity. For example, given a BER of $10^{-5}$, it reduces the detection candidate list generation complexity as well as the extrinsic LLR calculation complexity by a factor of 2 and 8 , respectively in an $(8 \times 4)$-element 4QAM system. The associated memory requirements were also reduced by a factor of 8 .

\section{REFERENCES}

[1] E. Viterbo and J. Boutros, "A universal lattice code decoder for fading channels," IEEE Transactions on Information Theory, vol. 45, pp. 16391642, July 1999.

[2] K. Wong, C. Tsui, R. S. K. Cheng, and W. Mow, "A VLSI architecture of a k-best lattice decoding algorithm for MIMO channels," Circuits and Systems, 2002. ISCAS 2002. IEEE International Symposium on, vol. 3, pp. 273-276, May 2002.

[3] L. Hanzo, M. Munster, B. J. Choi, and T. Keller, OFDM and MC-CDMA for Broadband Multi-User Communications, WLANs and Broadcasting. IEEE Press, 2003.

[4] T. Cui and C. Tellambura, "An efficient generalized sphere decoder for rank-deficient MIMO systems," 2004. VTC2004-Fall. 2004 IEEE 60th Vehicular Technology Conference, vol. 5, pp. 3689-3693, Sept. 2004.

[5] B. M. Hochwald and S. ten Brink, "Achieving near-capacity on a multipleantenna channel," IEEE Transactions on Communications, vol. 51, pp. 389-399, Mar. 2003.

[6] L. Hanzo, T. H. Liew, and B. L. Yeap, Turbo Coding, Turbo Equalisation and Space-Time Coding for Transmission over Fading Channels. IEEE Press, 2002.

[7] S. ten Brink, "Convergence behavior of iteratively decoded parallel concatenated codes," IEEE Transactions on Communications, vol. 49, pp. 1727-1737, Oct. 2001 